\title{
Échantillonnage et interprétation : application aux produits de saisie analysés par un laboratoire de toxicologie
}

\section{Sampling and interpretation : application to seized products analysed by a toxicology laboratory}

\section{Yacine AMRAOUI, Ingrid ALLIO, Carine GARCIA, Martine PERRIN*}

Institut de Recherche Criminelle de la Gendarmerie Nationale, 1, boulevard Théophile Sueur 93111 ROSNY-SOUS-BOIS Cedex

* Auteur à qui adresser la correspondance : Martine PERRIN, Institut de Recherche Criminelle de la Gendarmerie Nationale, 1, boulevard Théophile Sueur - 93111 ROSNY-SOUS-BOIS Cedex Tél : 0149355079 - Fax : 0149355027

(Reçu le 13 octobre 2001 ; accepté le 20 novembre 2001)

\section{$R E ́ S U M E ́$}

Lors d'une saisie de comprimés ou de toute autre population composée d'unités discrètes - (ie) que l'on peut dénombrer une certaine proportion de ces unités est susceptible de renfermer une substance classée sur la liste des stupéfiants. Pour des raisons évidentés de coût d'analyse et de faisabilité dans le temps, les laboratoires de criminalistique (gendarmerie, police, douanes, etc...) n'analysent qu'un échantillon de cette saisie, dont la taille variera selon la méthode employée. De nombreuses procédures d'échantillonnage sont utilisées à travers le monde. Elles peuvent sommairement être classées en deux catégories : d'une part celles s'appuyant sur des études empiriques, d'autre part celles reposant sur une approche statistique ou probabiliste. Les approches empiriques présentent notamment l'inconvénient d'induire une charge de travail excessive dès que la saisie devient importante ; quant à certaines approches statistiques, elles sont difficiles à appréhender par les non scientifiques. Cependant l'approche statistique permet de disposer d'une mesure scientifiquement "correcte" car elle a vocation

\section{SUMMARY}

Within a tablet seizure (or any population of discrete units), a certain proportion is likely to contain an illicit substance. For analysis cost or feasibility reasons, forensic laboratories analyse only a sample of the whole seizure, the size of which size may vary according to the sampling method employed. Many methods exist. They can be classified into two groups : empirical methods and methods based upon a statistic or a probabilistic approach. The first group leads to laboratory overwork, when a large amount of units are seized. Interpretation of statistical approaches can be delicate. Indeed, the scientist is looking for a clear theory accepted by court as an evidence, and which can be used to extrapolate the results of the sample analysis to the whole seizure or seal. In this context, the sampling method proposed by Colin Aitken (I) is particularly appropriate. It is based upon Bayes's subjective probability theory. Relatively simply to understand, it never leads to overwork. For instance, with a large consignment, using this method allows him to be $99 \%$ sure that the proportion of units containing something illegal 
d'indice pour l'enquêteur et l'expert et de preuve pour le magistrat requérant; elle doit aussi autoriser l'extrapolation des résultats de l'analyse de l'échantillon à l'ensemble du scellé ou de la saisie. Dans ce contexte, l'approche préconisée par Colin Aitken (1) est particulièrement adaptée. Elle se fonde sur la théorie des probabilités "subjectives" de Bayes. D'une grande lisibilité, elle n'aboutit jamais à une surcharge de travail et permet d'élaborer une stratégie d'échantillonnage convenant aux besoins de l'analyste et aux contraintes du laboratoire. Par exemple, pour des scellés de taille importante, il apparaîtra que l'échantillonnage de 6 unités, prises au hasard, dont l'analyse révèle qu'elles renferment toutes une substance inscrite sur la liste des stupéfiants, suffit pour affirmer qu'il y a $99 \%$ de chances que plus de la moitié des unités au sein du scellé renferment une substance illicite. Enfin, cette procédure est applicable à l'échantillonnage dans bien d'autres domaines (dès que le produit de l'analyse est composé d'unités discrètes) par exemple en criminalistique avec l'analyse de fausse monnaie, de supports multimédia à caractère pédophile..., mais aussi en analyse de contrôle de produit fini (médicaments, aliments...)...

\section{MOTS-CLÉS}

Échantillonnage, coût d'analyse, preuve, probabilités Baysiennes.

\section{Introduction}

Combien d'unités faut-il prélever pour obtenir un échantillon représentatif (c'est-à-dire un échantillon dont les caractéristiques sont aussi proches que possible de celles du scellé ou de la saisie), sans induire une trop grande charge de travail ? Tel est le problème auquel est confronté l'analyste. L'objectif de cet article, après un tour d'horizon critique des méthodes préconisées par diverses équipes au niveau international, est de proposer une procédure qui tienne compte des spécificités de fonctionnement des laboratoires de criminalistique.

\section{Conventions de langage}

Caractère étudié : présence d'une substance classée comme stupéfiant, telles qu'elles sont définies par l'arrêté du 22 février 1990 modifié.

$\mathrm{N}$ : taille de la population, c'est-à-dire nombre d'unités au sein du scellé.

$\mathrm{m}$ : taille de l'échantillon prélevé, c'est-à-dire nombre d'unités au sein de l'échantillon.

$\mathrm{n}$ : nombre d'unités n'ayant pas été échantillonnées. Conséquence : $\mathrm{N}=\mathrm{n}+\mathrm{m}$

$\mathrm{z}$ : nombre d'unités échantillonnées présentant le caractère étudié, c'est-à-dire renfermant une substance stupéfiante. $\mathrm{z}$ peut donc prendre les valeurs $0,1, \ldots, \mathrm{m}$.

$\theta$ : proportion d'unités au sein du scellé présentant le caractère étudié. is greater than 0,5, if the sampling of 6 units reveal they all contain something illegal. The method can have many other applications. In forensic sciences for example it can be applied to forgery (money), to multimedia supports which have a pedophilic content..., in the industrial field it can be applied to the quality check of production (pharmaceutical industry, food industry.......

\section{KEY-WORDS}

Sampling, analysis cost, evidence, Bayes's probabilities.

$\mathrm{p}$ : probabilité pour que la proportion d'unités au sein du scellé renfermant une substance stupéfiante soit strictement supérieure à $50 \%$.

$\mathrm{q}$ : probabilité pour que la proportion d'unités au sein du scellé renfermant une substance stupéfiante soit inférieure ou égale à $50 \%$.

$\mathrm{R}$ : variable aléatoire discrète, (c'est à dire prenant ses valeurs sur l'ensemble $\mathrm{N}$ des entiers naturels), et représentant le nombre total d'unités au sein du scellé présentant le caractère étudié.

$\mathrm{Z}$ : variable aléatoire discrète représentant le nombre total d'unités au sein de l'échantillon présentant le caractère étudié.

Y : variable aléatoire discrète représentant le nombre total d'unités au sein de la sous-population de taille $n$ n'ayant pas été échantillonnée présentant le caractère étudié. Conséquence : $\mathrm{R}=\mathrm{Z}+\mathrm{Y}$.

$\Theta$ : paramètre fixé appartenant à l'intervalle $[0 ; 1] .(\theta \neq \Theta)$ $\mathrm{P}(\mathrm{X}>\mathrm{x})$ : probabilité pour qu'une variable aléatoire $\mathrm{X}$ soit strictement supérieure à $\mathrm{X}$.

$\mathrm{IC}_{100(1-\alpha) \%}(\mathrm{X})$ : intervalle de confiance à $100(1-\alpha) \%$ pour une variable aléatoire $\mathrm{X}(\alpha \in[0 ; 1])$

$\mathrm{x} \rightarrow \mathrm{f}(\mathrm{x})$ : fonction qui à un réel $\mathrm{x}$ associe la quantité $\mathrm{f}(\mathrm{x})$. 


\section{Revue de littérature}

\section{Méthodes d'échantillonnage empiriques}

\section{Formules constantes}

Elles consistent à prélever un nombre fixe d'unités indépendamment de la taille du scellé. Il existe également des cas où le nombre d'unités à analyser est précisé par le magistrat (procureur de la république ou juge d'instruction). L'analyste doit alors se conformer à ses indications, bien qu'il soit du rôle de l'expert de "conseiller" le magistrat.

\section{Formule arithmétique}

$\mathbf{m}=\mathbf{2 0}+\mathbf{1 0 \%}(\mathrm{N}-20)(\forall \mathrm{N}>20)$

Cette formule est proposée par M. Colon et al. (2) et se base sur une étude empirique regroupant 173 saisies, soit un total de 19994 unités. A partir de ces données, les auteurs ont constaté que cette procédure permettait d'obtenir un échantillon représentatif.

Cette méthode présente l'inconvénient majeur d'introduire une relation croissante entre le nombre d'unités à échantillonner et la taille de la population. Ainsi, en présence d'un scellé de 500 unités, il est nécessaire d'en analyser environ 70 , ce qui aboutit à une charge de travail conséquente et donc inadaptée à la réalité d'un laboratoire.

\section{Formule des Nations Unies}

$m=N^{1 / 2}(\forall N>100)$

Cette formule préconisée par l'O.N.U.(programme des nations unies pour le contrôle international des drogues, division stupéfiants - Vienne)(2),(3) est destinée à l'usage des laboratoires nationaux d'analyse de stupéfiants et s'adapte à toute drogue saisie qui ne se présente pas en vrac (comprimés, doses d'héroïne de rue, pains de résines de cannabis...).

Cette méthode induit une forte charge de travail dès que la taille du scellé devient importante. De plus, à l'instar de la formule précédente, cette norme ne permet qu'une extrapolation empirique à l'ensemble du scellé des résultats de l'analyse de l'échantillon. Il existe enfin quelques variantes des deux formules ci-dessus présentant les mêmes difficultés(4).

\section{Méthodes statistiques d'échantillonnage} Méthode de Student (5)(6)

La méthode de Student, relative aux échantillons de petite taille, autorise à faire de l'inférence (c'est à dire à extrapoler) sur la teneur moyenne en substance stupéfiante au sein du scellé. Elle permet la construction d'un intervalle de confiance pour cette teneur moyenne, ou par analogie pour le poids total de drogue au sein du scellé. Elle présente également l'avantage de limiter la charge de travail et d'être valide d'un point de vue théorique si les hypothèses de base sont respectées.

A titre d'exemple, l'analyste prélève au hasard un échantillon de taille $\mathrm{m}$, pris parmi $\mathrm{N}$ unités. S'il procède à la quantification des stupéfiants présents dans chacune de celles-ci, il pourra aisément calculer la moyenne $\bar{M}$ des teneurs en stupéfiant au sein de l'échantillon. Le but est de construire un intervalle de confiance à $100(1-\alpha) \%$ pour la moyenne $M$ des teneurs en substance illicite au sein du scellé ( $\alpha \in[0 ; 1])$, c'est à dire un intervalle contenant la vraie moyenne $\mathrm{M}$ au risque de $100(1-\alpha) \%$. Pour ce faire, les deux variables suivantes sont introduites :

1) $s^{2}:$ variance empirique corrigée (non biaisée) telle que :

$\mathrm{S}^{2}=\frac{\sum_{i=1}^{m}\left(x_{i}-\bar{M}\right)^{2}}{m-1}$ où $\mathrm{x}_{i}$ désigne la teneur en subde l'échantillon $(i=1, \ldots, m)$.

2) $t$ : variable de Student telle que :

$$
\mathrm{t}=\frac{|\bar{M}-M|}{s} \sqrt{m}
$$

La table de Student indique les valeurs frontières que $\mathrm{t}$ a une probabilité donnée de dépasser.

Il faut alors distinguer deux cas.

Premier cas : $m / N<0,1$ :

Au niveau de confiance $100(1-\alpha) \%$, l'intervalle suivant est obtenu pour $\mathrm{M}$ :

$$
\mathrm{IC}_{100(1-\alpha) \%}(\mathrm{M})=\left[\bar{M} \pm \mathrm{t}_{m-1 ; \alpha / 2} \frac{s}{\sqrt{m}}\right]
$$

avec $\mathrm{m}-1$ : nombre de degrés de liberté, et $\alpha$, risque consenti.

Par exemple, pour $m=6$ et $\alpha=5 \%$, la valeur de $t$ lue dans la table est 2,57.

Second cas : $m / N \geq 0,1$ :

Il convient ici d'ajouter un facteur de correction. D'où l'intervalle suivant :

$$
\mathrm{IC}_{100(1-\alpha) \%}(\mathrm{M})=\left[\bar{M} \pm \mathrm{t}_{m-1 ; \alpha / 2} \frac{s}{\sqrt{m}} \sqrt{\frac{(N-m)}{N}}\right.
$$

Certes, cette méthode permet de limiter le nombre d'unités à prélever, néanmoins, faire de l'inférence sur cette teneur moyenne (ou sur un poids moyen de substance stupéfiante), est moins évocateur qu'une inférence $\operatorname{sur} \theta$. Dès lors, cette méthode pourra servir de complément à une étude statistique sur la proportion d'unités au sein du scellé renfermant une substance stupéfiante. 


\section{Loi hypergéométique (7)}

L'utilisation de cette loi est appropriée pour faire de l'inférence sur $\theta$. Deux approches sont possibles : la première en terme d'intervalle de confiance, la seconde en terme de tests entre hypothèses multiples.

La loi hypergéométrique permet en effet la construction d'un intervalle de confiance pour notre proportion $\theta$, sous trois conditions, relatives notamment aux tailles de la population et de l'échantillon. Pour construire un tel intervalle, il est nécessaire de raisonner avec une variable aléatoire continue (prenant ses valeurs sur l'ensemble $\mathrm{R}$ des nombres réels ou sur une partie de R), or la loi hypergéométrique est une loi discrète, c'est à dire prenant ses valeurs sur l'ensemble $\mathrm{N}$ des entiers naturels.

Il est donc nécessaire de procéder successivement à deux approximations qui permettront de raisonner avec une variable aléatoire suivant une loi continue (en l'occurrence la loi normale centrée) par application du théorème central limite. Ces deux approximations reposent sur la vérification des conditions évoquées cidessus. Mais celles-ci sont problématiques dans un tel contexte car elles nécessitent de travailler avec des échantillons de taille supérieure à 30 unités et en même temps d'avoir une population telle que $\mathrm{m} / \mathrm{N}<0,1$. De plus, la vérification de la troisième condition ne peut être évaluée qu'après l'analyse de l'échantillon. S'il s'avère qu'elle n'est pas remplie, les résultats de l'analyse ne permettront aucune extrapolation valide théoriquement. C'est pourquoi cette approche n'est guère satisfaisante.

Par contre, l'utilisation d'un test entre hypothèses multiples permettra de faire de l'inférence sur $\theta$ en utilisant la loi hypergéométrique (7). Cette méthode sera préconisée sur des populations dont la taille ne dépasse pas 50 unités. Dans le cas contraire, il faudra privilégier la loi binomiale (7).

Ainsi, le test suivant est introduit :

\section{Hypothèse dite nulle $(\mathrm{HO}): \theta>\Theta$ contre hypothèse dite alternative (H1) : $\theta \leq \Theta$}

Soit $\alpha$ la probabilité de commettre une erreur de première espèce lors de ce test, c'est-à-dire la probabilité de rejeter à tort l'hypothèse $\mathrm{H} 0$.

$(1-\alpha)$ pourra être exprimé comme une fonction de $\mathrm{N}$, $\mathrm{m}$, et $\Theta$ dans le cas notamment où toutes les unités échantillonnées présentent le caractère.

En outre, la borne inférieure $\Theta$ pourra être exprimée comme une fonction de $\mathrm{m}, \mathrm{N}$, du nombre d'unités échantillonnées ne présentant pas le caractère avec un niveau supérieur ou égal à $(1-\alpha)$.

Les difficultés liées à cette méthode sont dues à l'interprétation qui peut en résulter. Lorsqu'un test est construit, une quantité $\varepsilon$ appelée seuil du test est introduite $\left(\varepsilon \in \mathrm{R}^{+*}\right)$. Il faut alors imposer au test que la probabilité de commettre une erreur de première espèce soit toujours inférieure ou égale à $\varepsilon$, autrement dit : $\alpha<\varepsilon$. $\varepsilon$ doit être choisi très petit, par exemple $\varepsilon=0,01$ voire $\varepsilon=0,001$, en partant du principe que l'analyste croit fermement en l'hypothèse nulle (5).

Mais pour $\Theta=0,7, \mathrm{~N}=50, \mathrm{~m}=\mathrm{z}=5,(1-\alpha)=$ vaut 0,847 donc $\alpha>\varepsilon$.

Par contre, pour $\Theta=0,7, N=30, m=z=15,(1-\alpha)$ est proche de 1 , donc $\alpha<0,001$.

Dans ce dernier cas, l'interprétation est la suivante : "En présence d'une population de 30 unités dans laquelle est prélevé un échantillon de taille 15 dont toutes les unités présentent le caractère, rien ne s'oppose à admettre que $\theta$ soit strictement supérieur à $0,7 . "$.

Ce qui ne signifie pas que $\theta$ soit effectivement supérieur à 0,7 . De plus, la probabilité que $\theta$ soit supérieur à 0,7 n'est pas connue.

Dans les cas où $\alpha$ n'est pas suffisamment faible (strictement inférieur à 0,01 voire 0,001 selon la force du critère que l'analyste souhaite obtenir) rien ne permet réellement de trancher (4).

Cette méthode ne sera donc satisfaisante que dans les cas où la valeur de $\alpha$ - celle induite par l'expérience consistant à prélever parmi $\mathrm{N}$ unités $\mathrm{m}$ unités dont l'analyse révélera qu'elles sont au nombre de $\mathrm{z}$ à renfermer une substance stupéfiante - sera suffisamment faible et assortie d'une taille d'échantillon $m$ respectant les contraintes de charge de travail.

L'utilisation de la loi binomiale dans le cas de populations excédant 50 unités aboutit aux mêmes difficultés d'interprétation.

\section{Théorie des probabilités subjectives de Bayes(5)(8)}

Afin de pouvoir bénéficier de toute la flexibilité offerte par cette méthode, le laboratoire doit disposer d'un logiciel d'analyse numérique ou de calcul statistique approprié. La théorie Bayesienne permet alors de répondre - par exemple - à la question suivante : «quelle taille d'échantillon choisir pour être sûr à $100 \varphi \%$ que la proportion d'unités au sein du scellé renfermant une substance stupéfiante est strictement supérieure à $100 \Theta \%$ ? ( $\varphi$ et $\Theta$ appartenant à l'intervalle $[0,1]) »$.

Exemple : Pour $\varphi=0,99$ et $\Theta=0,5$ la question devient : quelle taille d'échantillon choisir pour être sûr à $99 \%$ que la proportion d'unités contenant une substance stupéfiante dépasse $50 \%$ ? 


\section{Descriptif de la méthode}

Cette méthode se base sur les présomptions de l'analyste concernant la vraie valeur de $\theta$, c'est à dire sur l'idée qu'il se fait de la vraie valeur de $\theta$ avant même d'avoir prélevé ou analysé l'échantillon. Cette présomption peut être le reflet de son expérience passée ou bien liée à un test qu'il aura effectué sur les unités de la saisie...

La méthode se réalise en trois étapes :

1/ Choix par l'analyste de deux constantes $\alpha$ et $\beta$ (appartenant à $\mathrm{R}^{+*}$ ) : Ces constantes reflètent la force de la présomption de l'analyste sur $\theta$, c'est à dire sur la proportion d'unités contenant un stupéfiant au sein de la saisie.

2/ Tirage aléatoire sans remise de m unités prises parmi $\mathrm{N}$ et analyse de l'échantillon ainsi constitué. L'analyste connaît alors le nombre $\mathrm{z}$ d'unités échantillonnées présentant le caractère étudié.

3/ Choix de $\varphi$ et $\Theta$ : l'analyste fixe ainsi une force de critère d'extrapolation et choisit la taille d'échantillon $m$ qui le satisfait sous l'hypothèse que $\mathrm{z}$ unités échantillonnées présentent le caractère. Concrètement, ce choix revient à résoudre une équation à l'aide d'un logiciel approprié. A l'issue de cette étude, l'analyste est en mesure d'affirmer qu'en prélevant un échantillon de taille $\mathrm{m}$ dont $\mathrm{z}$ unités présentent le caractère, il y a $100 \varphi \%$ de chances que $\theta$ soit strictement supérieur à $100 \Theta \%$.

Deux cas devront être distingués selon la taille du scellé considéré.

\section{Scellés de plus de 50 unités $(\mathbf{N}>\mathbf{5 0})$}

$\theta$ sera considéré comme une variable aléatoire continue à valeurs dans $] 0,1\left[\right.$ dont la densité de probabilité $\mathrm{f}_{\mathrm{a}}$ sera donnée par :

$$
\mathrm{f}_{a}(\theta / \alpha, \beta)=\frac{\theta^{\alpha-1}(1-\theta)^{\beta-1}}{B(\alpha, \beta)}(0<\theta<1)
$$

$$
\text { Avec }: \mathrm{B}(\alpha, \beta)=\frac{\Gamma(\alpha) \Gamma(\beta)}{\Gamma(\alpha+\beta)} \text { et } \Gamma(z)=\int_{0}^{+\infty} t^{z-1} e^{-t} d t
$$

$\mathrm{f}_{\mathrm{a}}(\theta / \alpha, \beta)$ se lit "densité antérieure de $\theta$ sachant $\alpha$ et $\beta^{\prime \prime}$. $\theta$ suit une loi Bêta de paramètre $\alpha$ et $\beta$ ( $\alpha$ et $\beta$ appartenant à $\mathrm{R}^{+*}$ ).

Cette densité a été indexée par la lettre "a" -"a" pour antérieur à l'analyse - car sa détermination dépend de la spécification avant tirage de l'échantillon des deux constantes $\alpha$ et $\beta$, dont le choix va refléter la présomption de l'analyste $\operatorname{sur} \theta$.

\section{Par exemple :}

- le choix de $\alpha=\beta=1$ appliqué à l'expression ci-dessus conduit à une densité de loi uniforme sur $[0,1]$, ce choix correspond donc au cas où l'incertitude de l'analyste concernant la vraie valeur de $\theta$ est maximale. Ceci signifie que le juge ou l'expert n'ont aucun a priori sur la composition de la saisie,

- le choix de $\alpha=3$ et $\beta=1$ reflète la présomption selon laquelle le résultat le plus probable est de voir toutes les unités de la saisie présenter le caractère,

- le choix de $\alpha=\beta=1 / 2$ reflète la présomption selon laquelle les 2 résultats suivants sont les plus probables: toutes les unités du scellé présentent le caractère ou au contraire aucune unité ne le présente, avec une croyance décroissant proportionnellement à $\mathrm{x} \rightarrow$ Arcsinus ( $2 x-1)$ quand $\theta$ parcourt $[0 ; 1]$ (à une constante près).

Pour visualiser la présomption induite par un choix donné de $\alpha$ et $\beta$, il faut pouvoir représenter graphiquement $\mathrm{f}_{\mathrm{a}}(\theta / \alpha, \beta)$ et éventuellement le complémentaire de la fonction de répartition associée.

Pour des valeurs entières de $\alpha$ et $\beta$, les calculs peuvent être effectués à l'aide d'une calculatrice scientifique. Par contre, pour des valeurs non entières de $\alpha$ et $\beta$, les calculs nécessitent l'utilisation d'un logiciel de calcul numérique ou statistique.

Bien entendu, il peut apparaître délicat pour l'analyste de formaliser ses présomptions et tout choix reste un acte subjectif. Ce choix devra par conséquent être justifié et il conviendra de trouver les valeurs de $\alpha$ et $\beta$ qui permettront de représenter cette présomption. Néanmoins, pour des valeurs de $\alpha$ et $\beta$ comprises entre 0 et 1 , des choix différents - (ie) des présomptions différentes - n'entraîneront pas de grandes différences au niveau des conclusions, ceci assurant une certaine stabilité à la méthode.

Il convient alors de combiner l'information fournie par la densité antérieure à celle inhérente à l'analyse qui révélera que $\mathrm{z}$ unité(s) parmi $\mathrm{m}$ renferment une substance stupéfiante. Cette dernière information est formalisée par une fonction de vraisemblance notée $\mathrm{L}$ ayant même forme mathématique que le germe d'une loi binomiale mais prenant $\mathrm{m}$ et $\mathrm{z}$ comme fixes et $\theta$ comme variable (contrairement à la loi binomiale). La densité postérieure au tirage notée $\mathrm{f}_{p}$ est alors obtenue. Elle est dite postérieure au tirage car elle ne peut être explicitée qu'une fois spécifiées les valeurs de $m$ et $z$. Cette vraisemblance $\mathrm{L}$ a la forme fonctionnelle suivante :

$$
\mathrm{L}(\theta)=C_{m}^{z} \theta^{z}(1-\theta)^{m-z}
$$

En combinant cette information à celle donnée par la densité antérieure et en vertu du théorème de Bayes, le résultat suivant est obtenu : 


$$
\mathrm{f}_{p}(\theta / \mathrm{z}, \mathrm{m}, \alpha, \beta)=\frac{\theta^{z+\alpha-1}(1-\theta)^{m-z+\beta-1}}{B(z+\alpha, m-z+\beta)}
$$

Lorsque l'analyste choisit $\varphi$ et $\Theta$ il doit résoudre pour $\mathrm{m}$ l'équation suivante :

$\mathrm{P}(\theta>\Theta / z, \mathrm{~m}, \alpha, \beta)=\varphi$

Ce qui revient à résoudre :

$\mathrm{P}(\Theta<\theta<1 / \mathrm{z} \mathrm{m}, \alpha, \beta)=\varphi$

$\Leftrightarrow \int_{\Theta}^{1} f_{p}(\theta / z, m, \alpha, \beta) d \theta=\varphi$

La résolution s'opère par un processus de tâtonnement qui consiste à choisir "au hasard" une taille d'échantillon $\mathrm{m}$, à vérifier si cette valeur satisfait ou non l'équation ci-dessus ; si ce n'est pas le cas, il conviendra d'ajuster la valeur de $m$ jusqu'à vérification de cette équation.

Les tableaux I, III et $\mathrm{V}$ résument les résultats respectivement pour $\alpha=\beta=1 ; \alpha=3, \beta=1$ et $\alpha=\beta=1 / 2$. Les résultats seront commentés dans la section suivante.

\section{Saisies de moins de 50 unités $(\mathbf{N} \leq \mathbf{5 0})$}

La population étant de petite taille, une meilleure représentation de la variabilité du nombre d'unités non examinées présentant le caractère sera obtenue en considérant explicitement une loi de probabilité pour ce nombre $\mathrm{Y}$, inconnu mais inférieur ou égal à $n$.

Étant donné $\theta$, la distribution de $(\mathrm{Y} / \mathrm{n}, \theta)$ et de $(\mathrm{Z} / \mathrm{m}$, $\theta$ ) est une loi binomiale, $\theta$ suit une loi Bêta.

Les distributions $(Y / n, \theta)$ et $(\theta / m, z, \alpha, \beta)$ peuvent être combinées pour obtenir une distribution prédictive Bayesienne pour $(\mathrm{Y} / \mathrm{m}, \mathrm{n}, \mathrm{z}, \alpha, \beta)$ en appliquant un raisonnement analogue à celui exposé précédemment.

Dans le cas général ( $\alpha$ et $\beta$ quelconques), le résultat suivant est obtenu :

$\mathrm{P}(\mathrm{Y}=\mathrm{y} / \mathrm{m}, \mathrm{n}, \mathrm{z}, \alpha, \beta)=\frac{\Gamma(m+\alpha+\beta) C_{n}^{y} \Gamma(m+n-z-y+\beta)}{\Gamma(z+\alpha) \Gamma(m-z+\beta) \Gamma(m+n+\alpha+\beta)}$

Dans le cas où $\alpha=\beta=1$, ceci devient :

$$
\mathrm{P}(\mathrm{Y}=\mathrm{y} / \mathrm{m}, \mathrm{n}, \mathrm{z}, 1,1)=\frac{(m+1) C_{m}^{*} C_{n}^{\nu}}{(m+n+1) C_{m+n}^{++y}}
$$

\section{Exemple avec $\alpha=\beta=1$}

10 unités sont prélevées au sein d'un scellé de taille 30 . L'analyste constate, après identification et quantification, que 9 unités sur les 10 présentent le caractère étudié.

Quelle est la probabilité pour que $\theta$ soit supérieur ou égal à $80 \%$ ? Autrement dit, que vaut la quantité $\mathrm{P}(\theta \geq 0,8)$ ?

Ici, $\mathrm{N}=30, \mathrm{~m}=10, \mathrm{n}=20$ et $\mathrm{z}=9$.

Pour que $\theta$ soit supérieur ou égal à 0,8 il faut et il suffit que le scellé renferme au moins 24 unités présentant le caractère. L'analyse ayant révélé que 9 unités contenaient une substance stupéfiante, il faut et il suffit que le nombre Y soit supérieur ou égal à $15(15+9=24)$. En termes probabilistes, ceci correspond au fait que les deux événements aléatoires suivants sont équivalents : $\{\theta \geq 0,8\}$ et $\{\mathrm{Y}=15\} \cup\{\mathrm{Y}=16\} \cup\{\mathrm{Y}=17\} \cup$ $\{\mathrm{Y}=18\} \cup\{\mathrm{Y}=19\} \cup\{\mathrm{Y}=20\}$

Il convient alors de remarquer que la réunion d'événements ci-dessus concerne des événements disjoints, c'est à dire qui ne peuvent se réaliser en même temps. Sachant que deux événements équivalents ont une même probabilité d'occurrence, le résultat suivant est obtenu :

$$
\mathrm{P}(\theta \geq 0,8)=\mathrm{P}(\mathrm{Y} \geq 15)=\sum_{k=15}^{20} P(Y=k / 10,20,9,1,1)
$$

$\Rightarrow \mathrm{P}(\theta \geq 0,8)=\sum_{15}^{20} \frac{11^{*} C_{10}^{9} C_{20}^{k}}{31^{*} C_{30}^{9+K}} \approx 80 \%$

(en remplaçant $\mathrm{m}, \mathrm{z}, \mathrm{n} \alpha$ et $\beta$ par leur valeur d'expérience)

Ainsi, en présence d'un scellé de taille 30, d'un échantillon de 10 unités dont 9 présentent le caractère, l'analyste est en mesure d'affirmer qu'il y a $80 \%$ de chances qu'il y ait au moins $80 \%$ d'unités contenant une substance stupéfiante au sein du scellé considéré, avec $\alpha=\beta=1$.

\section{Commentaires des résultats}

\section{Cas où $\alpha=\beta=1$}

Ces résultats sont valables quand l'analyste ne peut a priori se prononcer sur la vraie valeur de $\theta$ (incertitude maximale), ou que le juge n'émet aucune hypothèse en fonction des éléments du dossier.

\section{$\mathbf{N}>\mathbf{5 0}$}

Le tableau I indique qu'en prélevant un échantillon de 6 unités dont toutes renferment une substance illicite, il y a $99 \%$ de chances que la proportion d'unités au sein du scellé considéré présentant le caractère soit strictement supérieure à 0,5 ; il y a donc $99 \%$ de chances que plus de la moitié des unités du scellé renferment une substance stupéfiante $(m=6, z=6, p=0,99)$. Si parmi les 6 unités prélevées, une et une seule ne renferme rien d'illégal, cette probabilité est alors de $93 \%(\mathrm{~m}=6, \mathrm{z}=5$, $\mathrm{p}=0,93$ ). Si l'analyste souhaite à nouveau obtenir une certitude de $99 \%$, il peut prélever 4 unités supplémentaires. Si toutes les 4 présentent le caractère, $\mathrm{P}(\theta>0,5)$ $=99 \%(\mathrm{~m}=10, \mathrm{z}=9, \mathrm{p}=0,99)$. Si parmi ces 4 unités prélevées en second lieu une et une seule ne renferme aucune substance illicite, $\mathrm{P}(\theta>0,5)=96 \%(\mathrm{~m}=10$, $\mathrm{z}=8, \mathrm{p}=0,96)$. 
Les valeurs mentionnées dans chacun des tableaux ne tiennent pas compte des règles d'arrondis (arrondi au chiffre inférieur). L'hypothèse la plus favorable à la défense est ainsi privilégiée.

Tableau I : Probabilité pour que plus de la moitié des unités au sein du scellé présentent le caractère étudié en fonction de la taille d'échantillon $(m)$ et du nombre d'unités échantillonnées renfermant une substance classée comme stupéfiant (z), avec $N>50$ et $a=b=1$.

\begin{tabular}{|c|c|c|}
\hline $\mathbf{m}$ & $\mathbf{z}$ & $\mathbf{p}$ \\
\hline 6 & 6 & 0,99 \\
\hline 6 & 5 & 0,93 \\
\hline 6 & 4 & 0,77 \\
\hline 6 & 3 & 0,5 \\
\hline 6 & 2 & 0,22 \\
\hline 6 & 1 & 0,06 \\
\hline 6 & 0 & 0,007 \\
\hline 10 & 9 & 0,99 \\
\hline 10 & 8 & 0,96 \\
\hline 10 & 7 & 0,88 \\
\hline 10 & 6 & 0,72 \\
\hline 10 & 5 & 0,5 \\
\hline
\end{tabular}

\section{$\mathbf{N} \leq \mathbf{5 0}$}

Le tableau II indique par exemple qu'en présence d'un scellé de 30 unités, si sur 5 unités échantillonnées toutes renferment une substance illicite, il y a $99 \%$ de chances que $\theta$ soit supérieur ou égal à $0,5(\mathrm{~N}=30, \mathrm{~m}=5, \mathrm{z}=5$, $q=0,99)$. Si parmi ces 5 unités prélevées, une et une seule ne renferme rien d'illicite $\mathrm{P}(\theta \geq 0,5)=92 \%(\mathrm{~N}=$ $30, \mathrm{~m}=5, \mathrm{z}=4, \mathrm{q}=0,92$ ). De même, si l'analyste souhaite obtenir une certitude de $99 \%$, il peut prélever 3 unités supplémentaires; si toutes les 3 contiennent une substance stupéfiante $\mathrm{P}(\theta \geq 0,5)=99 \%(\mathrm{~N}=30, \mathrm{~m}=$ $8, \mathrm{z}=7, \mathrm{q}=0,99$ ). Si parmi ces 3 unités prélevées en second lieu une et une seule ne renferme rien d'illicite, $P(\theta \geq 0,5)=95 \%(N=30, m=8, z=6, q=0,95) . S i$ parmi ces 3 unités prélevées en second lieu exactement 2 d'entre elles ne renferment rien d'illégal $\mathrm{P}(\theta \geq 0,5)=$ $81 \%(\mathrm{~N}=30, \mathrm{~m}=8, \mathrm{z}=5, \mathrm{q}=0,81)$.

En cas de scellés de taille intermédiaire (par exemple $\mathrm{N}$ $=24$ ), l'analyste pourra ici utiliser les résultats correspondant à $\mathrm{N}=30$. La probabilité pour que la proportion soit supérieure ou égale à 0,5 sera alors supérieure ou égale à la valeur correspondant à $\mathrm{N}=30$ avec les mêmes valeurs pour $m$ et $z$.

\section{Cas où $\alpha=3$ et $\beta=1$}

Ces résultats sont valables dans le cas où l'analyste pense a priori que le résultat le plus probable est que toutes les unités au sein du scellé renferment une substance illicite.
Tableau II : Probabilité pour que au moins la moitié des unités au sein du scellé présentent le caractère étudié en fonction de la taille du scellé $(N)$, de la taille d'échantillon (m) et du nombre d'unités échantillonnées renfermant une substance classée comme stupéfiant (z), avec $N \leq 50$ et $a=b=1$.

\begin{tabular}{|c|c|c|c|c|c|c|c|c|}
\hline $\mathbf{N}$ & \multicolumn{7}{|c|}{10} \\
\hline $\mathbf{m}$ & 4 & 4 & 4 & 4 & 4 & 6 & 6 & 6 \\
\hline $\mathbf{z}$ & 4 & 3 & 2 & 1 & 0 & 5 & 4 & 3 \\
\hline $\mathbf{P}(q \geq \mathbf{0 . 5})$ & 0,99 & 0,93 & 0,60 & 0,17 & 0,01 & 1 & 0,95 & 0,65 \\
\hline
\end{tabular}

\begin{tabular}{|c|c|c|c|c|c|c|c|c|c|}
\hline $\mathbf{N}$ & \multicolumn{10}{|c|}{20} \\
\hline $\mathbf{m}$ & 5 & 5 & 5 & 5 & 5 & 5 & 7 & 7 & 7 \\
\hline $\mathbf{z}$ & 5 & 4 & 3 & 2 & 1 & 0 & 6 & 5 & 4 \\
\hline $\mathbf{P}(\mathrm{q} \geq \mathbf{0 . 5})$ & 0,99 & 0,94 & 0,73 & 0,36 & 0,09 & 0,008 & 0,99 & 0,93 & 0,73 \\
\hline
\end{tabular}

\begin{tabular}{|c|c|c|c|c|c|c|c|c|c|c|}
\hline $\mathbf{N}$ & \multicolumn{10}{|c|}{30} \\
\hline $\mathbf{m}$ & 5 & 5 & 5 & 5 & 5 & 5 & 8 & 8 & 8 & 8 \\
\hline $\mathbf{z}$ & 5 & 4 & 3 & 2 & 1 & 0 & 7 & 6 & 5 & 4 \\
\hline $\mathbf{P}(\mathrm{q} \geq \mathbf{0 . 5})$ & 0,99 & 0,92 & 0,70 & 0,35 & 0,09 & 0,01 & 0,99 & 0,95 & 0,81 & 0,54 \\
\hline
\end{tabular}

\begin{tabular}{|c|c|c|c|c|c|c|c|c|c|c|}
\hline $\mathbf{N}$ & \multicolumn{10}{|c|}{40} \\
\hline $\mathbf{m}$ & 5 & 5 & 5 & 5 & 5 & 5 & 8 & 8 & 8 & 8 \\
\hline $\mathbf{z}$ & 5 & 4 & 3 & 2 & 1 & 0 & 7 & 6 & 5 & 4 \\
\hline $\mathbf{P}(\mathrm{q} \geq \mathbf{0 . 5})$ & 0,99 & 0,91 & 0,69 & 0,35 & 0,10 & 0,01 & 0,99 & 0,94 & 0,79 & 0,53 \\
\hline
\end{tabular}

\begin{tabular}{|c|c|c|c|c|c|c|c|c|c|c|}
\hline $\mathbf{N}$ & \multicolumn{10}{|c|}{50} \\
\hline $\mathbf{m}$ & 5 & 5 & 5 & 5 & 5 & 5 & 8 & 8 & 8 & 8 \\
\hline $\mathbf{z}$ & 5 & 4 & 3 & 2 & 1 & 0 & 7 & 6 & 5 & 4 \\
\hline $\mathbf{P}(\mathbf{q} \geq \mathbf{0 . 5})$ & 0,99 & 0,91 & 0,68 & 0,35 & 0,10 & 0,01 & 0,99 & 0,93 & 0,78 & 0,52 \\
\hline
\end{tabular}

\section{$\mathbf{N}>\mathbf{5 0}$}

Le tableau III indique qu'en prélevant un échantillon de 4 unités dont toutes renferment une substance illicite, il y a $99 \%$ de chances que $\theta$ soit strictement supérieur à $0,5(\mathrm{~m}=4, \mathrm{z}=4, \mathrm{p}=0,99)$. Lorsque une ou plusieurs unités échantillonnées ne renferment rien d'illicite, les résultats sont interprétés comme précédemment, c'està-dire en comptant le nombre total d'unités ayant été échantillonnées $(\mathrm{m})$ et le nombre total d'unités échantillonnées présentant le caractère $(\mathrm{z})$.

\section{$\mathbf{N} \leq \mathbf{5 0}$}

Le tableau IV indique par exemple qu'en présence d'un scellé de 40 unités dans lequel est prélevé un échantillon de taille 5 dont toutes les unités renferment une substance illicite, il y a $99 \%$ de chances que $\theta$ soit supérieur ou égal à $0,5(\mathrm{~N}=40, \mathrm{~m}=5, \mathrm{z}=5, \mathrm{q}=0,99)$. 
Tableau III : Probabilité pour que plus de la moitié des unités au sein du scellé présentent le caractère étudié en fonction de la taille d'échantillon $(m)$ et du nombre d'unités échantillonnées renfermant une substance stupéfiante (z), avec $N>50 ; a=3$ et $b=1$.

\begin{tabular}{|c|c|c|}
\hline $\mathbf{m}$ & $\mathbf{z}$ & $\mathbf{p}$ \\
\hline 4 & 4 & 0,99 \\
\hline 4 & 3 & 0,93 \\
\hline 4 & 2 & 0,77 \\
\hline 4 & 1 & 0,50 \\
\hline 4 & 0 & 0,22 \\
\hline 8 & 7 & 0,99 \\
\hline 8 & 6 & 0,96 \\
\hline 8 & 5 & 0,88 \\
\hline 8 & 4 & 0,72 \\
\hline 8 & 3 & 0,5 \\
\hline
\end{tabular}

Tableau IV : Probabilité pour que au moins la moitié des unités au sein du scellé présentent le caractère étudié en fonction de la taille du scellé $(N)$, de la taille d'échantillon (m) et du nombre d'unités échantillonnées renfermant une substance stupéfiante (z), avec $N \leq 50 ; a=3$ et $b=1$.

\begin{tabular}{|c|c|c|c|c|c|c|c|}
\hline $\mathbf{N}$ & \multicolumn{6}{|c|}{10} \\
\hline $\mathbf{m}$ & 4 & 4 & 4 & 4 & 4 & 4 & 5 \\
\hline $\mathbf{z}$ & 4 & 3 & 2 & 1 & 0 & 4 & 3 \\
\hline $\mathbf{P}(q \geq \mathbf{0 . 5})$ & 0,99 & 0,97 & 0,79 & 0,38 & 0,07 & 0,99 & 0,91 \\
\hline
\end{tabular}

\begin{tabular}{|c|c|c|c|c|c|c|c|c|}
\hline $\mathbf{N}$ & \multicolumn{7}{|c|}{20} \\
\hline $\mathbf{m}$ & 4 & 4 & 4 & 4 & 4 & 6 & 6 & 6 \\
\hline $\mathbf{z}$ & 4 & 3 & 2 & 1 & 0 & 5 & 4 & 3 \\
\hline $\mathbf{P}(q \geq \mathbf{0 . 5})$ & 0,99 & 0,95 & 0,77 & 0,44 & 0,14 & 0,99 & 0,93 & 0,75 \\
\hline
\end{tabular}

\begin{tabular}{|c|c|c|c|c|c|c|c|c|c|c|}
\hline $\mathbf{N}$ & \multicolumn{10}{|c|}{30} \\
\hline $\mathbf{m}$ & 4 & 4 & 4 & 4 & 4 & 6 & 7 & 7 & 7 & 7 \\
\hline $\mathbf{z}$ & 4 & 3 & 2 & 1 & 0 & 5 & 6 & 5 & 4 & 3 \\
\hline $\mathbf{P}(q \geq \mathbf{0 . 5})$ & 0,99 & 0,94 & 0,77 & 0,46 & 0,17 & 0,98 & 0,99 & 0,96 & 0,84 & 0,60 \\
\hline
\end{tabular}

\begin{tabular}{|c|c|c|c|c|c|c|c|c|c|c|}
\hline $\mathbf{N}$ & \multicolumn{10}{|c|}{40} \\
\hline $\mathbf{m}$ & 5 & 5 & 5 & 5 & 5 & 5 & 6 & 7 & 7 & 7 \\
\hline $\mathbf{z}$ & 5 & 4 & 3 & 2 & 1 & 0 & 5 & 6 & 5 & 4 \\
\hline $\mathbf{P}(\mathbf{q} \geq \mathbf{0 . 5})$ & 0,99 & 0,97 & 0,86 & 0,62 & 0,32 & 0,10 & 0,98 & 0,99 & 0,95 & 0,84 \\
\hline
\end{tabular}

\begin{tabular}{|c|c|c|c|c|c|c|c|c|c|c|}
\hline $\mathbf{N}$ & \multicolumn{10}{|c|}{50} \\
\hline $\mathbf{m}$ & 5 & 5 & 5 & 5 & 5 & 5 & 6 & 7 & 7 & 7 \\
\hline $\mathbf{z}$ & 5 & 4 & 3 & 2 & 1 & 0 & 5 & 6 & 5 & 4 \\
\hline $\mathbf{P}(q \geq \mathbf{0 . 5})$ & 0,99 & 0,97 & 0,86 & 0,62 & 0,33 & 0,11 & 0,98 & 0,99 & 0,95 & 0,83 \\
\hline
\end{tabular}

\section{Cas où $a=b=1 / 2$}

Ces résultats sont valables dans le cas où l'analyste pense a priori que les deux résultats suivants sont les plus probables :

- toutes les unités du scellé présentent le caractère,

- aucune unité ne le présente.

\section{$\mathbf{N}>\mathbf{5 0}$}

Le tableau $\mathrm{V}$ indique qu'en prélevant un échantillon de 5 unités dont toutes renferment une substance illicite, il y a $99 \%$ de chances que q soit strictement supérieur à $0,5(\mathrm{~m}=5, \mathrm{z}=5, \mathrm{p}=0,99)$.

\section{$\mathbf{N} \leq \mathbf{5 0}$}

Le tableau VI indique par exemple qu'en présence d'un scellé de 20 unités dans lequel est prélevé un échantillon de 4 unités dont toutes renferment une substance illicite, il y a $99 \%$ de chances que q soit supérieur ou égal à $0,5(\mathrm{~m}=4, \mathrm{z}=4, \mathrm{q}=0,99)$.

Tableau V : Probabilité pour que plus de la moitié des unités au sein du scellé présentent le caractère étudié en fonction de la taille d'échantillon $(m)$ et du nombre d'unités. échantillonnées renfermant une substance stupéfiante (z), avec $N>50 ; a=b=1 / 2$.

\begin{tabular}{|c|c|c|}
\hline $\mathbf{m}$ & $\mathbf{z}$ & $\mathbf{p}$ \\
\hline 5 & 5 & 0,99 \\
\hline 5 & 4 & 0,91 \\
\hline 5 & 3 & 0,66 \\
\hline 5 & 2 & 0,33 \\
\hline 5 & 1 & 0,08 \\
\hline 5 & 0 & 0,006 \\
\hline 6 & 5 & 0,95 \\
\hline 7 & 6 & 0,97 \\
\hline 8 & 7 & 0,98 \\
\hline 9 & 8 & 0,99 \\
\hline 9 & 7 & 0,95 \\
\hline 9 & 6 & 0,84 \\
\hline 9 & 5 & 0,62 \\
\hline 9 & 4 & 0,34 \\
\hline
\end{tabular}


Tableau VI : Probabilité pour que au moins la moitié des unités au sein du scellé présentent le caractère étudié en fonction de la taille du scellé (N), de la taille d'échantillon (m) et du nombre d'unités échantillonnées renfermant une substance stupéfiante (z), avec $N \leq 50 ; \alpha=\beta=1 / 2$.

\begin{tabular}{|c|c|c|c|c|}
\hline $\mathbf{N}$ & \multicolumn{4}{|c|}{10} \\
\hline $\mathbf{m}$ & 3 & 3 & 3 & 3 \\
\hline $\mathbf{z}$ & 3 & 2 & 1 & 0 \\
\hline $\mathbf{P}(\theta \geq \mathbf{0 . 5})$ & 0,99 & 0,81 & 0,32 & 0,02 \\
\hline
\end{tabular}

\begin{tabular}{|c|c|c|c|c|c|}
\hline $\mathbf{N}$ & \multicolumn{5}{|c|}{20} \\
\hline $\mathbf{m}$ & 4 & 4 & 4 & 4 & 4 \\
\hline $\mathbf{z}$ & 4 & 3 & 2 & 1 & 0 \\
\hline $\mathbf{P}(\theta \geq \mathbf{0 . 5})$ & 0,99 & 0,89 & 0,54 & 0,15 & 0,01 \\
\hline
\end{tabular}

\begin{tabular}{|c|c|c|c|c|c|c|}
\hline $\mathbf{N}$ & \multicolumn{6}{|c|}{30} \\
\hline $\mathbf{m}$ & 5 & 5 & 5 & 5 & 5 & 5 \\
\hline $\mathbf{z}$ & 5 & 4 & 3 & 2 & 1 & 0 \\
\hline $\mathbf{P}(\theta \geq \mathbf{0 . 5})$ & 0,99 & 0,94 & 0,71 & 0,34 & 0,08 & 0,004 \\
\hline
\end{tabular}

\begin{tabular}{|c|c|c|c|c|c|c|}
\hline $\mathbf{N}$ & \multicolumn{6}{|c|}{40} \\
\hline $\mathbf{m}$ & 5 & 5 & 5 & 5 & 5 & 5 \\
\hline $\mathbf{z}$ & 5 & 4 & 3 & 2 & 1 & 0 \\
\hline $\mathbf{P}(\theta \geq \mathbf{0 . 5})$ & 0,99 & 0,93 & 0,70 & 0,34 & 0,08 & 0,005 \\
\hline
\end{tabular}

\begin{tabular}{|c|c|c|c|c|c|c|c|}
\hline $\mathbf{N}$ & \multicolumn{7}{|c|}{50} \\
\hline $\mathbf{m}$ & 6 & 6 & 6 & 6 & 6 & 6 & 6 \\
\hline $\mathbf{z}$ & 6 & 5 & 4 & 3 & 2 & 1 & 0 \\
\hline $\mathbf{P}(\theta \geq \mathbf{0 . 5})$ & 0,99 & 0,96 & 0,82 & 0,52 & 0,20 & 0,04 & 0,002 \\
\hline
\end{tabular}

\section{Avantages de la méthode et arguments pouvant être présentés à l'audience}

L'approche Bayesienne permet la construction d'un critère lisible, y compris aux yeux de personnes n'ayant pas de connaissances en statistiques .

Par ailleurs, cette méthode est caractérisée par une grande flexibilité car le choix de $\alpha$ et $\beta$ permet de refléter la présomption du juge, de l'enquêteur ou de l'expert sur la composition de la saisie. Ces valeurs pourront varier d'une saisie à l'autre ou d'un laboratoire à l'autre.

Une objection pertinente visant à remettre en cause la méthode consisterait à dire que rien ne prouve que les unités n'ayant pas été échantillonnées présentent le caractère. Effectivement, le critère énoncé est une probabilité. S'il y a $90 \%$ de chances qu'un événement se réalise, rien ne permet d'affirmer qu'il se réalisera. Mais émettre une telle objection revient à omettre la nature des notions probabilistes et la portée pouvant leur être prêtée.

Un exemple concret peut venir étayer cette affirmation. Soit un scellé de 100000 unités, dans lequel est prélevé un échantillon de taille $\mathrm{m}=6$. Après analyse, il apparaît que les six unités présentent le caractère.

Supposons que l'avocat de la défense réfute la méthode employée en prétextant que les unités échantillonnées par l'analyste pourraient très bien être les seules à contenir une substance illégale.

Pour répondre à cette objection, il suffit d'utiliser la loi hypergéométrique. En effet, en faisant l'hypothèse que la population de 100000 unités ne renferme que 6 unités illicites, il est possible de calculer la probabilité de tirer sans remise un échantillon de taille $m=6$ dont toutes les unités présentent le caractère. Soit $\mathrm{P}$ cette probabilité. Il apparaît que :

$$
\mathrm{P}=\frac{C_{6}^{6} C_{99994}^{0}}{C_{100000}^{6}}=7,2.10^{-28} \approx 0 .
$$

Cela est donc quasiment impossible.

En faisant varier le nombre supposé d'unités présentant le caractère au sein du scellé, l'analyste pourra également énoncer à l'audience une série de probabilités représentant la vraisemblance de telles objections. Il suffira pour cela de considérer différents seuils, en fonctions de N. Par exemple, pour une population de taille $\mathrm{N}=1000$, le même raisonnement qui ci-dessus sera effectué, puis le nombre $\mathrm{k}$ supposé d'unités présentant le caractère au sein de la population sera progressivement augmenté $(k=50,100, \ldots 1000)$, et pour chaque valeur de $\mathrm{k}$ la probabilité de tirer sans remise une main de 6 unités présentant toutes le caractère sera calculée.

Afin de garantir la validité théorique de cette méthode, il convient de respecter le caractère aléatoire du tirage. Celui-ci doit être effectué indépendamment de toute considération (caractéristiques physiques des unités par exemple).

\section{Conclusion}

La réalisation d'un plan d'échantillonnage respectant les contraintes de charge de travail des laboratoires est un problème récurrent, qui peut être résolu par diverses méthodes dont la plus satisfaisante reste l'utilisation de la théorie des probabilités subjectives de Bayes, telle qu'elle est préconisée par Aitken (1).

Cette théorie est la seule permettant d'obtenir un canevas de réflexions, satisfaisant d'un point de vue intuitif pour des intervenants non statisticiens et en adéquation 
avec les spécificités des laboratoires de criminalistique. La procédure décrite ci-dessus sera intégrée à la stratégie analytique du département toxicologie de l'institut de recherche criminelle de la gendarmerie nationale.

En outre, cette méthode peut être complétée par la construction d'un intervalle de confiance pour la teneur moyenne en substance illicite ou pour le poids total de drogue au sein de la saisie en utilisant la méthode de Student.

Enfin, cette procédure est applicable à l'échantillonnage dans d'autres domaines dès que la saisie ou le scellé sont composés d'unités discrètes (fausse monnaie, contrefaçon, support multimédia à caractère pédophile...).

\section{Références}

1. Aitken C.G.G., Sampling - How big a sample ? J. Forensic Sci. 1999 ; 44 (4) : 750-760.

2. P.N.U.C.I.D. (programme des Nations Unies pour le contrôle international des drogues). Méthodes recommandées pour l'identification de l'amphétamine et de la methamphétamine (manuel à l'usage des laboratoires nationaux de stupéfiants), document référencé ST/NAR/9, V88-20134, février 1988 - Nations Unies (Autriche).

3. U.N.D.C.P. (United Nations drug control protocol) Recommended methods for testing illicit ring-substituted amphetamine derivatives (manual for use by national narcotics laboratories) ST/NAR/12, V93-85932, juin 1993, Nations Unies (Autriche).

4. Colon M., Rodriguez G., Diaz R.O. Representative Sampling of "Street" Drug Exhibits. J. Forensic Sci. 1993 ; 38 (3) : 641-648.

5. Jaffard P. Initiation aux méthodes de la statistique et du calcul des probabilités. In : Masson ed. Paris, 1996 ; 179 180.

6. Miller J., Statistics and chemometrics for analytical chemistry, 4th edition, Pearson education limited, Harlow, 2000.

7. Tzidony D., Ravreby M. Statistical Approach to Drug Sampling : A Case Study. J. Forensic Sci. 1992 (6); 37 : 1541-1549.

8. Aitken $\mathrm{C}$, Bring $\mathrm{J}$, Leonard $\mathrm{T}$, Papasouliotis $\mathrm{O}$. Estimation of quantities of drugs handled and the burden of proof. J. R; Statist. Soc., 1997; 160 (2) : 333-350. 